Document downloaded from:

http://hdl.handle.net/10251/60647

This paper must be cited as:

Bosch Roig, I.; Gómez, S.; Vergara Domínguez, L. (2011). A ground system for early forest fire detection based on infrared signal processing. International Journal of Remote Sensing. 32(17):4857-4870. doi:10.1080/01431161.2010.490245.

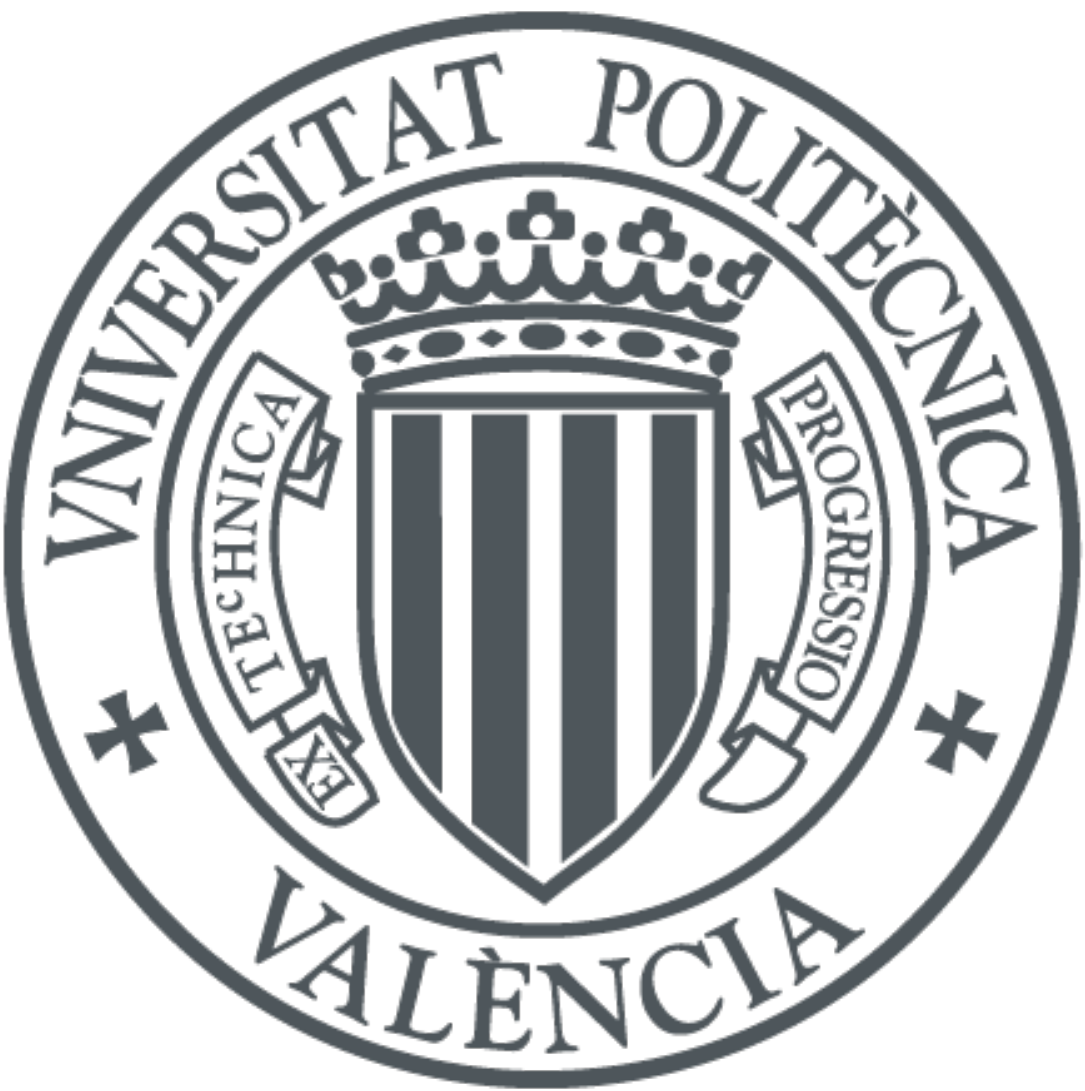

The final publication is available at

http://dx.doi.org/10.1080/01431161.2010.490245

Copyright Taylor \& Francis

Additional Information 


\title{
A ground system for early forest fire detection based on infrared signal processing
}

\author{
I. BOSCH$\dagger^{*}$, S. GÓMEZ†, L. VERGARA† \\ $\dagger$ Signal Processing Group, Institute of Telecommunications and Multimedia applications \\ (ITEAM), Polytechnic University of Valencia, Camino de Vera S/N 46022, Valencia, \\ Spain
}

\begin{abstract}
This paper presents a ground remote automatic system for forest surveillance based on infrared signal processing applied to early fire detection. Advanced techniques, which are based on infrared signal processing, are used in order to process the captured images. With the aim of determining the presence or absence of fire, the system performs the fusion of different detectors that exploit different expected characteristics of a real fire, such as persistence and increase. Theoretical simulations and practical results are presented to corroborate the control of the probability of false alarm. Results in a real environment are also presented to authenticate the accuracy of the operation of the proposed system. In particular, some experiments have been done to evaluate the delay of the system (tens of seconds on average) in detecting a controlled ground fire in range between $1 \mathrm{~km}$ and $10 \mathrm{~km}$. Moreover temporary evolution of false alarms and true detections are presented to evaluate the long-term performance of the system in a real environment. We have reached a detection probability of $100 \%$ at a false alarm rate of around $1 \times 10^{-9}$.
\end{abstract}

Keywords: forest fire detection, infrared signal processing, and decision fusion

\section{Introduction}

The objective of this proposal is to design and introduce a forest fire detection system that makes the early detection of such disasters possible. The main purpose of systems of this kind is to reduce the associated ecologic and economic consequences. This type of system could be integrated within a global system of emergency management to not only preserve the flora and fauna but also to complement other basic lines of action such as safety.

The early detection of forest fires is extremely important for the protection and conservation of natural spaces and is one of the great, challenges and obligations of fire managers and government agencies in the current society. Fire is one of the main enemies of these natural spaces (Pastor et al. 2003).

\footnotetext{
*Corresponding author. Email: igbosroi@dcom.upv.es
} 
This technology reduces human limitations and helps protect areas that otherwise would be difficult to protect. This technology facilitates the use of advanced systems that allow for efficient monitoring of extensive areas, regardless of the atmospheric conditions or the time of day.

One possibility is the use of satellite sensors, but they have limited spatial and temporal resolutions, which is why these systems are not able to detect early forest fires in a specific zone within seconds. Other options are the use of distributed systems on the ground, which are based on detection by images using diverse techniques of image processing directly on visual images (Dedouglu et al. 2005, Li et al. 2005) and infrared (IR) technology, which has been used by a few scientific groups (Arrue et al. 2000, Vicente and Guillemant 2002, Briz et al. 2003). In these works, signal processing algorithms are calibrated in a rather empirical manner, without relating to optimum filtering or detection theory.

With respect to the above works using infrared signals, we have introduced some significant novelties in the proposed processing scheme. Essentially, the algorithms are selected inside the framework of optimum statistical signal processing theory. Optimality is in the sense of the Neyman-Pearson criterion: given a selected Probability of False Alarm (PFA), try to maximize the Probability of Detection (PD). This is the most appropriate approach in this case, where a priori probability of the presence of an uncontrolled fire is very small. Hence, two main problems must be solved. First, we must be able to control the PFA, second, the detector should maximize the PD. In our scheme, we have total control of the PFA because we have knowledge of the statistical distribution of every statistic involved in it. Also the PD is maximized by appropriate selection of different energy detectors, which are known to be optimum under very general models of the background noise and of the unknown signal to be detected.

Moreover, the processing scheme is able to reject false detections not only from the background noise but also from specific events like cars or sudden weather changes. This is achieved by the combined effect of what we call persistence and increase detectors, which respectively exploit short-term and long-term effects of an uncontrolled fire. Thus, the overall final PFA is kept under control even in the presence of such undesired events.

Furthermore, additional improvement of the PD (and, thus, of the sensitivity of the system to the presence of a true fire) is obtained by means of a preprocessing step, which takes advantage of the stationary behavior of the infrared noise background that comes from the area under surveillance. This is achieved by means of a predictor, which reduces the noise background level. This improves the Signal to Noise Ratio (SNR) at the input of the detector subsystem, thereby increasing the PD.

In summary, the proposed system provides total control of a tolerable level of false alarms and has maximum sensitivity to the presence of an uncontrolled fire for the defined falsealarm rate. In the end, this implies a greater detection range and greater reliability of the overall system.

In this paper, we propose an automatic ground-based remote system for wide-area fire detection. Specifically, we present a system based on the use of thermal images. The different system blocks for the capture and processing of images are presented in the diagram depicted in Figure 1. The IR images captured from infrared cameras at local stations are processed in real time to minimize the information flow and to meet temporal restrictions, therefore, only the coordinates of the fire alarms are sent to the central station, 
where they are plotted on a map zone by means of the Geographic Information System (GIS).

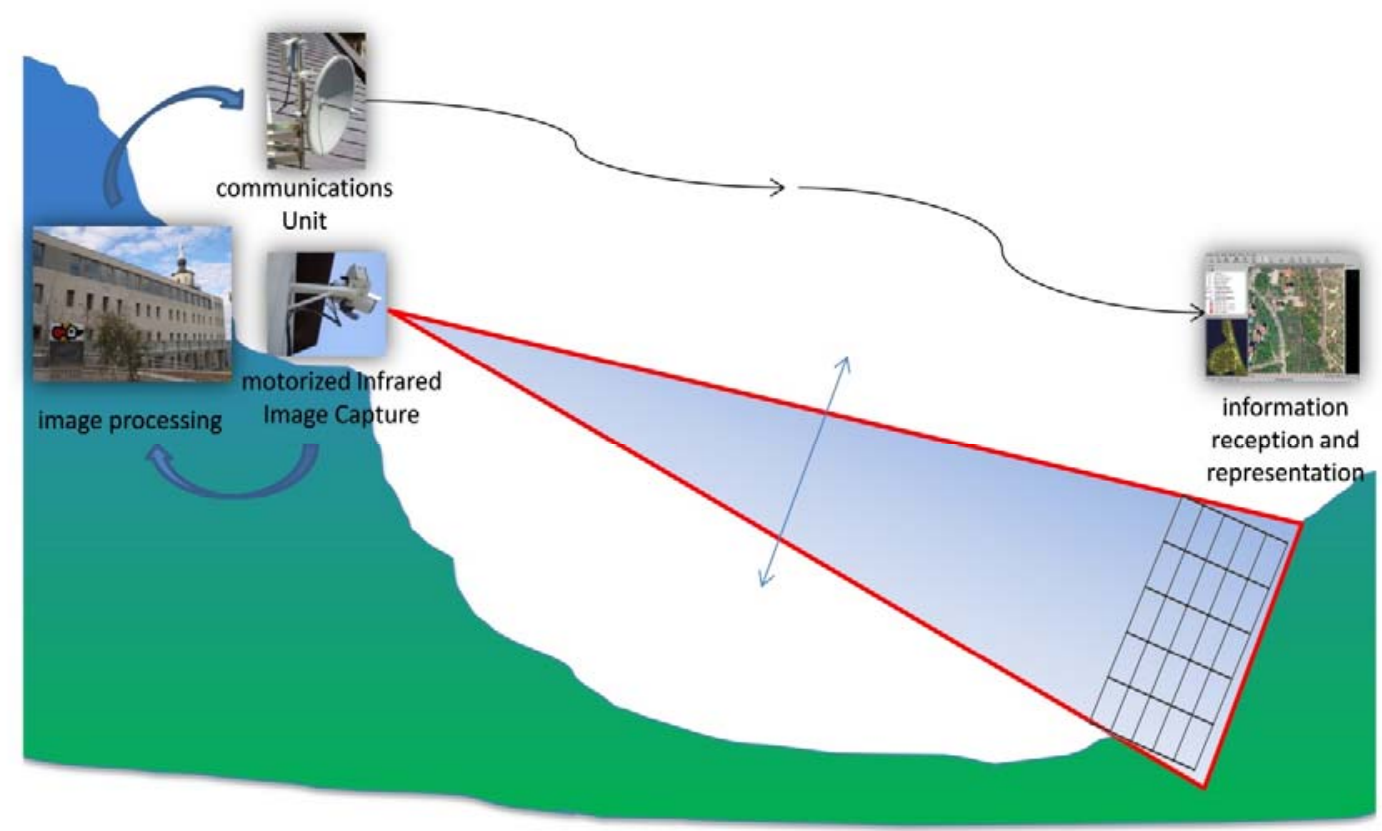

Figure 1: Global system diagram with thermal images processed and sent to the GIS

\section{Local Station Functionality}

The main functions of the local station are data acquisition and real-time image processing. In addition, a communication unit manages the information transmission between the local and the central stations. The description of the different subsystems is given below.

\subsection{Image Capture}

As stated in the introduction, local stations are based on the use of several low-cost infrared cameras, which are strategically placed to render a required coverage. In an attempt to improve this coverage, camera motorization and orographic studies could be used to eliminate redundancy in the covered area, thereby minimizing the number of sensors required and defining their optimal location.

An acquisition card captures the images from the IR sensor. The captured images will be the input data to the image-processing step. Furthermore, another camera in the video range is used to provide visible access to the area under surveillance. For example, if an alarm is detected, the system captures both thermal image and a visible one, which only contributes to information if there is sufficient diurnal light. In spite of the automatic operation of the 
system, both cameras can be remotely controlled in manual mode in order to change system parameters or to verify the detected fire threats.

The model of infrared camera used in this application was the A20V by FLIR Systems, whose characteristics are the following: field of view $25^{\circ} \times 19^{\circ}$; thermal sensitivity $0.12^{\circ}$ (at $30^{\circ} \mathrm{C}$ ); spatial resolution $160 \times 120$ pixels; and spectral range $7.5-13 \mu \mathrm{m}$. This is a lowcost camera without the capacity to show temperature values. However, this does not imply any limitations for the developed algorithms since, they do not require the specific values of temperature, but rather the relative increments of infrared energy in every pixel with respect to some properly defined reference level. In any case, this is not a problem since IR sensors can calculate surface temperature with the use of certain algorithms. Moreover, the proposed system indirectly uses physical properties of the environment (such as temperature) to reduce false alarms. In the calibration stage, the system adjusts the sensitivity based on numerical parameters such as level and span, which are supplied by the infrared camera. These have a direct correspondence with physical temperature values.

The cameras are largely protected from inclement weather by means of protective housing, which is mounted on a continuous motor. This motor is programmed to continuously sweep the covered zone. In spite of this, under certain extreme conditions (low-lying clouds, fog, or mist), the system may not be able to penetrate them and would not work correctly.

\subsection{Image Processing}

Once the thermal images have been captured, as described above, the most important stage of the system begins: the image processing stage.

In order to have automatic alarm detection without the need for human intervention, a class approach is to process each image separately by simply using the energy level from each pixel with respect to a predetermined threshold, as a reference. This threshold is suitably fixed to satisfy a certain probability of false alarm (PFA) obtaining a probability of detection (PD) that depends on the signal to noise ratio (SNR).

Nevertheless, in order to increase the processing sensitivity, we have decided to take advantage of the high existing correlation between successive images, when there are no abrupt changes of the environmental conditions or any fires. We perform a pixel to pixel processing, taking as a reference the latest and most recent images since the temperature for the same resolution cell throughout successive images, changes slowly over time.

In order to do this, we turn each thermal image into a matrix of pixels, where each pixel is associated with a resolution cell corresponding to certain coordinates of rank and azimuth. In this way, we generate a vector that describes the time history of each resolution cell (see Figure 2). 


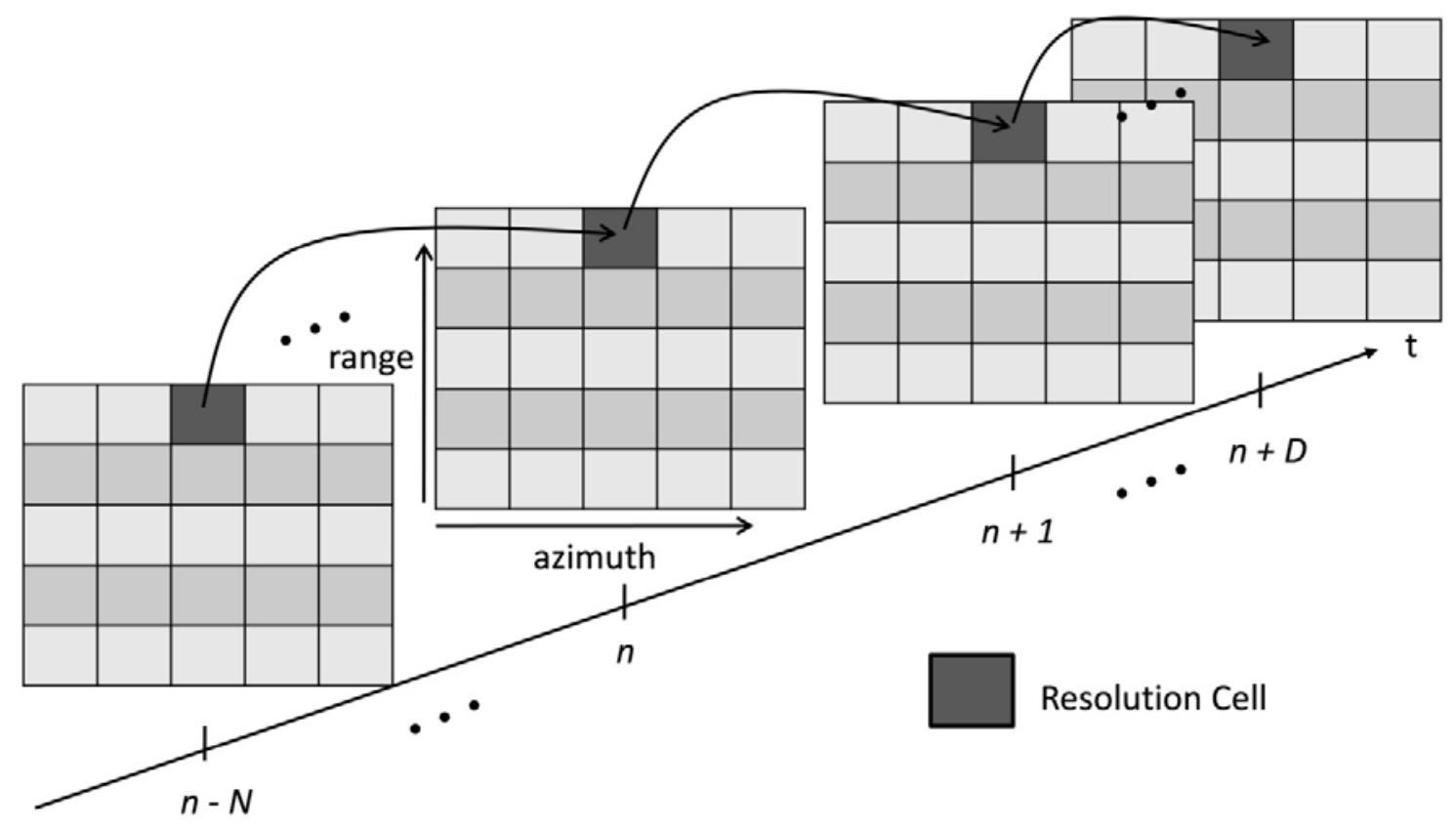

Figure 2: Temporal processing of resolution cells, pixel by pixel.

\subsubsection{General Detector}

The algorithm used in the improved system is based on the general scheme of Figure 3, where the general detector is applied to each resolution cell, which makes use of two kinds of detectors. Thus, several matched subspace detectors corresponding to short-term intervals are fused to obtain the so-called persistence decision. Then a long-term increase detector is implemented to obtain the increase decision. Finally, both decisions are fused, as shown in Figure 4. 


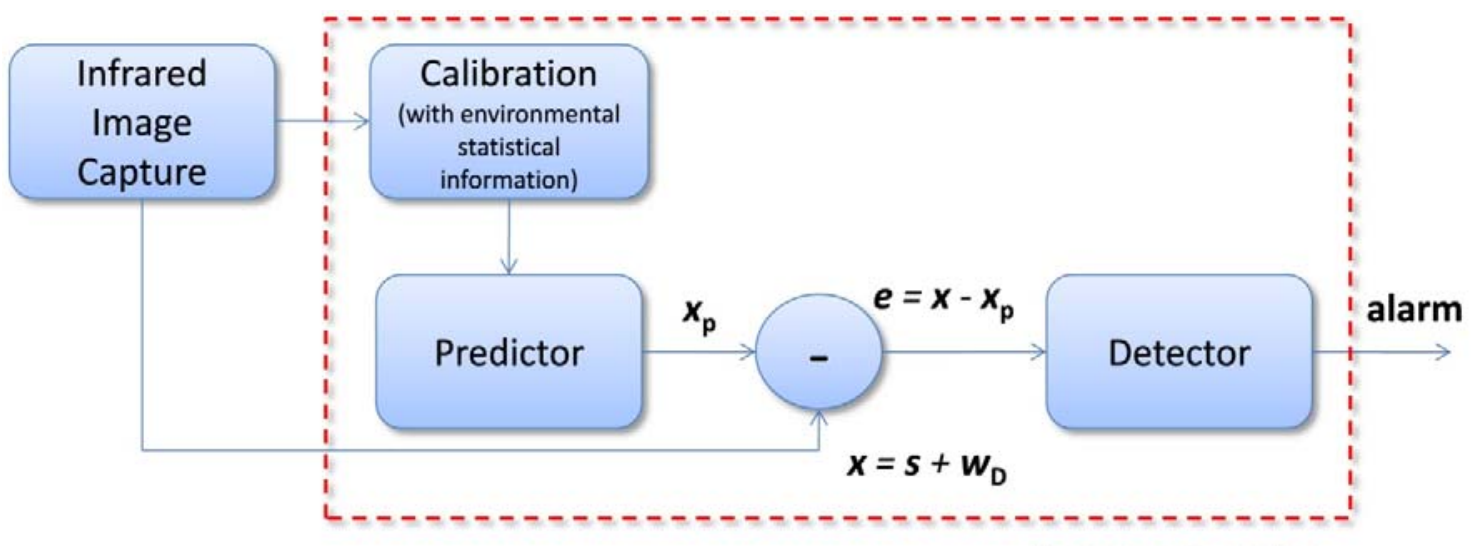

Image processing

Figure 3. General scheme of an uncontrolled fire detector.

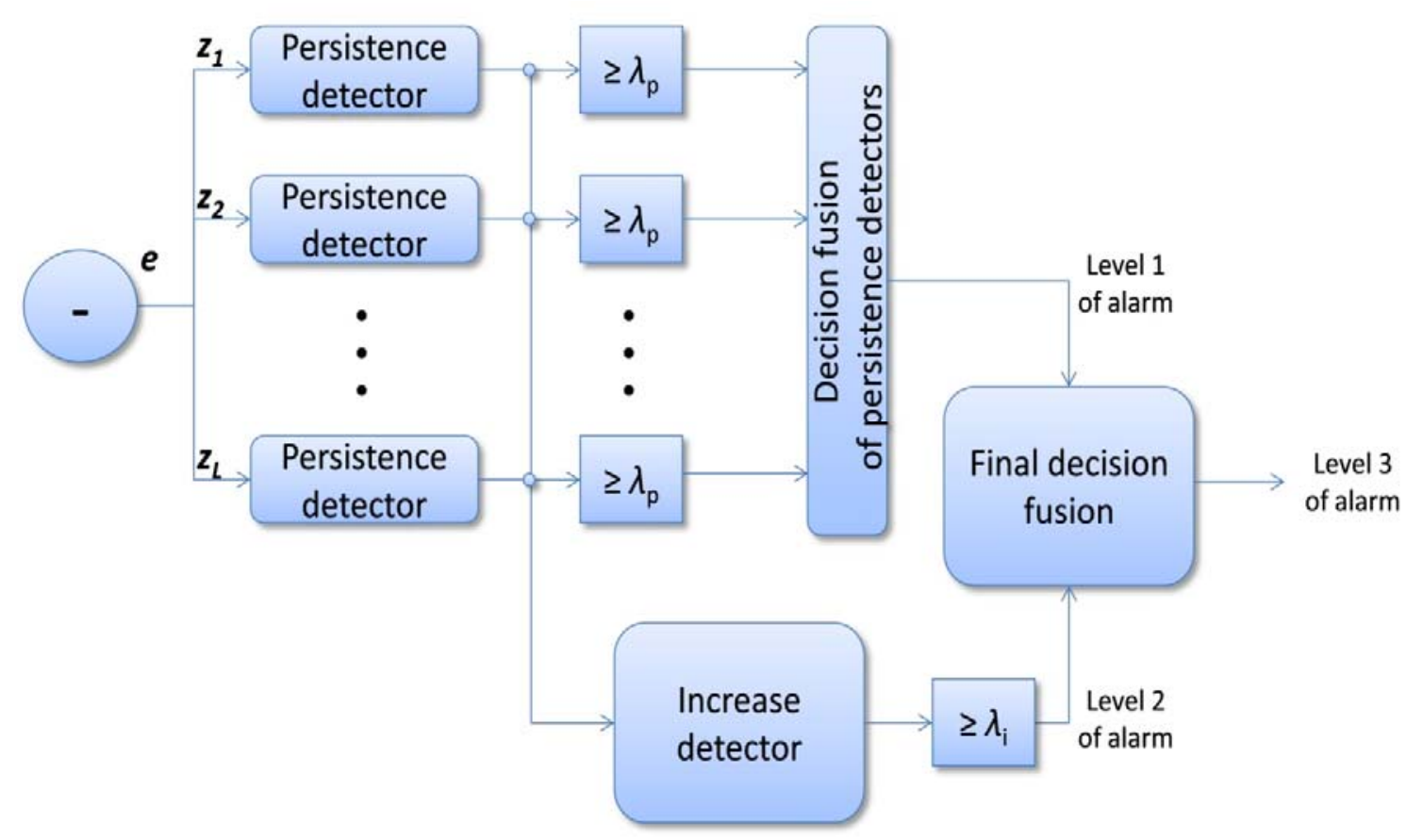

Figure 4. Global Detector scheme with persistence and increase detectors and final fusion rule

Some previous works by the authors have led to the scheme that was finally implemented in the system. Thus, the optimum design of a linear predictor was considered for the first time for this application in Vergara and Bernabeu (2000). It was then extended to a nonGaussian case by including a nonlinear gain in Vergara and Bernabeu (2001). The noise prediction computes the noise level of the cell being tested, using different scans. Then, this 
estimated level is subtracted from the pixel being tested, therely improving SNR. Note that if the SNR is increased a better PD is obtained for a given PFA.

Also, in Vergara and Bernabeu (2000), a matched subspace detector was considered to suppress undesired alarms due to "occasional" effects like cars, people, or wind. Bernabeu et al. (2004) complemented the matched subspace detector with an increase detector to take advantage of the expected increasing temperature behaviour of an uncontrolled fire. Fusion of the decisions made by the two detectors was also considered by Bernabeu et al. (2004). Finally, the complete detection algorithm was described in Bosch and Vergara, (2006), but considering a different time-scale for each detector.

A main objective is to have control of the overall PFA; hence, in Bosch et al. (2007), presented extensive simulations to verify the capability of controlling the overall PFA in the complete detector system. We show new results corresponding to the real operation of the implemented system in section 3.

As the general scheme of Figure 3 shows, the input to the detector is the level of one resolution cell through $D$ consecutive infrared image scans, which is named vector $\boldsymbol{x}=\boldsymbol{s}+\boldsymbol{w}_{\mathbf{D}}$. This vector is made up of a noise component $\boldsymbol{w}_{\mathbf{D}}$ and a signal component $\boldsymbol{s}$ due to the possible presence of fire when a true alarm is present.

In order to improve significantly the SNR, the predictor $\boldsymbol{x}_{\mathbf{p}}$ is introduced in the detector (the optimum predictor was designed by Vergara and Bernabeu (2000)). This reference signal $\boldsymbol{x}_{\mathbf{p}}$ is based on $N$ consecutive scans of the cell being tested, which is obtained in the calibration of the system, assuming that there is no signal present in those scans. Then the prediction $\boldsymbol{x}_{\mathbf{p}}$ is subtracted from the signal $\boldsymbol{x}$ to get the detector input $\boldsymbol{e}$.

Finally, Figure 4 summarizes the detection stage of the detection. This stage operates under the premise that an uncontrolled fire is a fire that persists over time and whose temperature continuously increases. This contrasts with other occasional effects like cars or atmospheric changes that may produce undesired alarms.

We have designed two types of detectors: short-term and long-term, adapted to each one of the fire premises. $L$ persistence short-term detectors are fused to generate a first-level alarm. The energy statistics that correspond to each persistence detector are joined together into a single vector, which is the input to the long-term increase detector. This generates a second-level alarm. Finally, the persistence and increase decisions are fused to generate a third and the most important alarm level.

In this paper, we only present a brief description of these detectors since a detailed study of each one can be found in the references mentioned above.

\subsubsection{Persistence Detector}

The first category of detector is the chain of persistence detectors that allows false alarms to be eliminated due to sporadic effects whose behavior can be assumed to be "high pass". Therefore, the persistence detector is implemented by dividing vector $\boldsymbol{e}$ into $L$ nonoverlapped segments $\mathbf{z}_{1} \ldots \mathbf{z}_{L}$, assuming that the fire signature across each segment is inside a "low pass" subspace with projection matrix $\mathbf{P}$.

The energy $E_{i}$, inside the subspace $\mathbf{P}$, of vector $\mathbf{z}_{\boldsymbol{i}}$, is compared with a threshold $\lambda$ that is calculated to meet a desired PFA $\left(P_{\mathrm{f} 0}\right)$, which is the probability of false alarm for each 
individual detector (which is equal for all of the chain) in the form of a classic subspace matched filter, and has a chi-square distribution function with $p$ degrees of freedom.

$$
E_{i}=\mathbf{z}_{i}^{T} \mathbf{P} \mathbf{z}_{i<}^{>} \lambda
$$

The other important parameter is the false alarm probability after decision fusion, $P_{\mathrm{fp}}$. The optimal decision fusion and the expression for $P_{\mathrm{fp}}$, considering independent decisions, is given by Bosch and Vergara (2006)

$$
P_{\mathrm{f} p}=\sum_{k=v+1}^{L}\left(\begin{array}{l}
L \\
K
\end{array}\right)\left(P_{\mathrm{f} 0}\right)^{k}\left(1-\left(P_{\mathrm{f} 0}\right)\right)^{L-k}+\gamma\left(\begin{array}{l}
L \\
v
\end{array}\right)\left(P_{\mathrm{f} 0}\right)^{k}\left(1-\left(P_{\mathrm{f} 0}\right)\right)^{L-k}
$$

Bosch et al. (2007) show the dependence of $P_{\mathrm{fp}}$ on the parameters involved ( $L, v$ and $\left.P_{\mathrm{f} 0}\right)$ by means of simulations. A main conclusion is that $P_{\mathrm{fp}}$ can be easily controlled by properly selecting the values $L, v$ and $P_{\mathrm{f}}$, thus validating equation (2). Being the threshold of the decisions fusion of persistence detectors, $v$, the number of persistence detectors that we demand for taking the final decision.

\subsubsection{Increase Detector}

The second category of detector is the increase detector that enhances the presence of increasing trends. The detector is based on a filter that is matched to a continuous component, which is preceded by a linear transformation $\mathbf{Q}^{(\mathrm{n})}$ whose order depends on the speed at which the fire increases. It is implemented by looking for increasing trends in the energy vector $\mathbf{z}_{\mathbf{E}}=\left[E_{1} \ldots E_{L}\right]^{\mathrm{T}}$ where $E_{i}$ is defined in equation (1). To this end, the vector $\mathbf{z}_{E}$ is transformed by a difference matrix of order $n, \mathbf{Q}^{(\mathrm{n})}$, and then matched to a continuous component vector $\boldsymbol{S}_{n}=[1 \ldots 1]^{\mathrm{T}}$, with $L-n$ ones. The corresponding test is given by equation (3) as shown in Bosch and Vergara (2006):

$$
\frac{\mathbf{z}_{\mathbf{E}}{ }^{\mathrm{T}}\left(\mathbf{Q}^{(n)}\right)^{\mathrm{T}}\left(\mathbf{Q}^{(n)}\left(\mathbf{Q}^{(n)}\right)^{\mathrm{T}}\right)^{-1} \boldsymbol{s}_{n}}{\sqrt{2 p \mathbf{s}_{n}^{\mathrm{T}}\left(\mathbf{Q}^{(n)}\left(\mathbf{Q}^{(n)}\right)^{\mathrm{T}}\right)^{-1} \boldsymbol{s}_{n}}}>\lambda_{0}
$$

where threshold $\lambda_{0}$ is calculated to meet a desired PFA called $P_{\text {fi. }}$.

\subsubsection{Final Decision}

The third and last detector is the final decision rule that was formulated in Bosch and Vergara (2006) as follows: 


$$
R(\boldsymbol{u})=\left\{\begin{array}{l}
1, \text { if } \boldsymbol{u}=\left[\begin{array}{ll}
1 & 1
\end{array}\right] \\
0, \text { otherwise }
\end{array}\right.
$$

where $\boldsymbol{u}=\left[u_{p} u_{i}\right]^{\mathrm{T}}$ are the binary random variables that represent the persistence and increase decisions, respectively.

The optimal decision fusion and the expression for $P_{\mathrm{ft}}$, assuming independence between the increase and persistence detector, is:

$$
P_{\mathrm{ft}}=P_{\mathrm{fp}} P_{\mathrm{fi}}
$$

Bosch et al. (2007) also showed the dependence of $P_{\mathrm{ft}}$ on $L$ and $P_{\mathrm{fo}}$ and the possibility to obtain similar theoretical and experimental results, thorough simulation, thus validating equation (5)

\subsection{Communication Unit}

Once the captured infrared images are processed, the information from the detected alarms of the local station is sent to the central station. Therefore, the communication unit performs the management for a correct transmission of this basic information. The type of transmission system that is suitable is determined by the amount of information to be transmitted, the orographic information, and the locations of the local and central stations. For example, the system implemented and described in section 3 consists of a $2.45 \mathrm{GHz}$ Wireless-LAN network system mounted on an open source software platform (Linux) that allows the acquisition, positioning, processing, local archiving, and connection between the local and the central station as well as between the central station and an internet web server. Nevertheless, other configurations may be applicable, and the system design must be implemented according to the specific problem and its location characteristics.

If a possible fire is detected, an alarm must be sent to the central station indicating the geographic location of the fire in minimum time and optimizing the communication resources. To avoid an excessive flow of information, only alarm threat coordinates, together with complementary time-space information, are sent from the local station to the central station.

In addition, an optimized system of information compression has been implemented to transmit only the contour of the form of the object described by the alarms, when they are numerous and close to each other. In other words, this optimized system allows the transmission of information from the pixels that have optimally generated each alarm. If the alarm only affects a small number of pixels, each one of the pixels is transmitted with its own level of alarm. However, if the alarm affects a group of pixels that suggests a certain form, then the contour of the form is transmitted to indicate that this group of pixels should be deal, with in a different way, for example, by filling in the inside of the contour.

Alarms from different local stations are processed and presented in the central computer. Therfore, each alarm is represented in the correct geographic coordinates using a 
Geographic Information System (GIS). The central computer also controls the different remote stations. The central station information is accessible via web (see Figure 5).

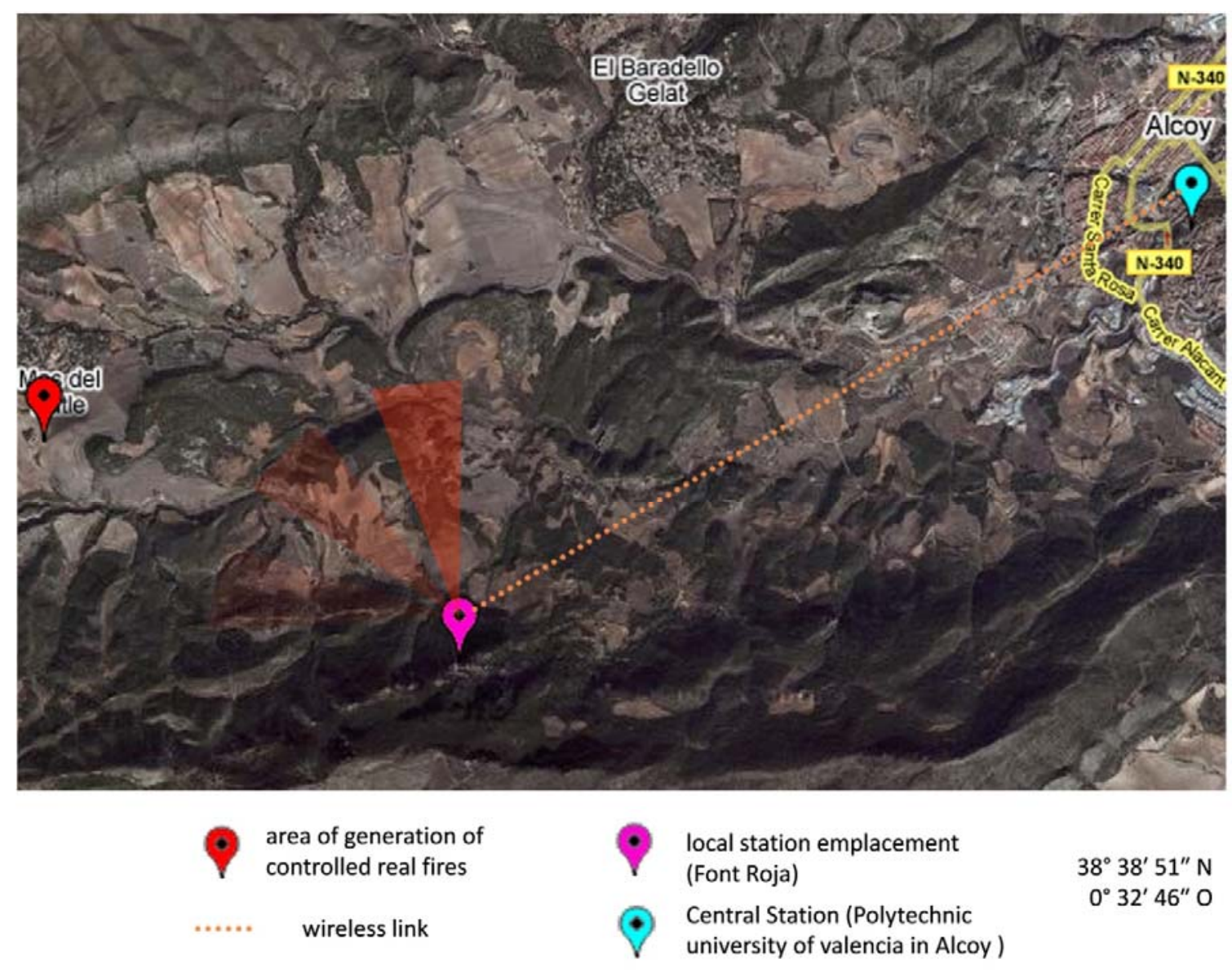

Figure 5. Location of central and local stations.

The central computer also performs other functions, such us management of historic alarm data-bases or remote connection to surveillance ports, (i.e., access alarm software visualization or to focus a camera in a particular direction by means of motorization).

In addition, all the control parameters of the system can be modified in real time by the central station through of communications unit for example, some of these parameters are: the identifier of each camera to indicate if there is more than one system; the specific zone of pixels to process in each image, allowing the undesired zones to be omitted; the number of captured images to calibrate each preset; the number of images used in the increase detector $(L)$; the number of processes (without alarm) that is needed to perform a new 
calibration in order to pass to the following preset; the number of previous images $(N)$ and future images $(D)$ required by the processing algorithms; the number of motor presets; the different controls of maximum time between calibrations, captures, processing, etc.; the communication ports used for the communication with the IR camera and with the motor; etc.

Finally, note that the system has control of the thresholds of each detector type, which are calculated from the desired PFA and from the sensitivity of the system, which depends on the level and the span obtained from the camera in each recalibration. This allows the thresholds to change based on the different seasons and the different weather conditions, such as morning, afternoon, night, summer, winter, etc.

\section{Results in a Real Environment}

Once the design of the system was developed, some experiments were performed to demonstrate the correct operation of the proposed system in a real environment.

The system was installed in Alcoy (Valencia, Spain), in the natural park named "El Carrascal de la Font Roja". The Font Roja natural park area is about 2500 hectares, and its maximum altitude is 1352 meters. Specifically, the system was installed in the "Sanctuary of the Red Font" (see Figure 1). This building is located in a privileged place at the top of the mountain providing a detailed view of the closest valley, which is oriented towards the northecist as shown in Figure 5.

This privileged location made the need for a Digital Elevation Model (DEM) of the forest area under control unnecessary. Usually, forests that are located in hilly terrain with shadows require the use of a clear model of the region to suitably locate each one of the cameras. 


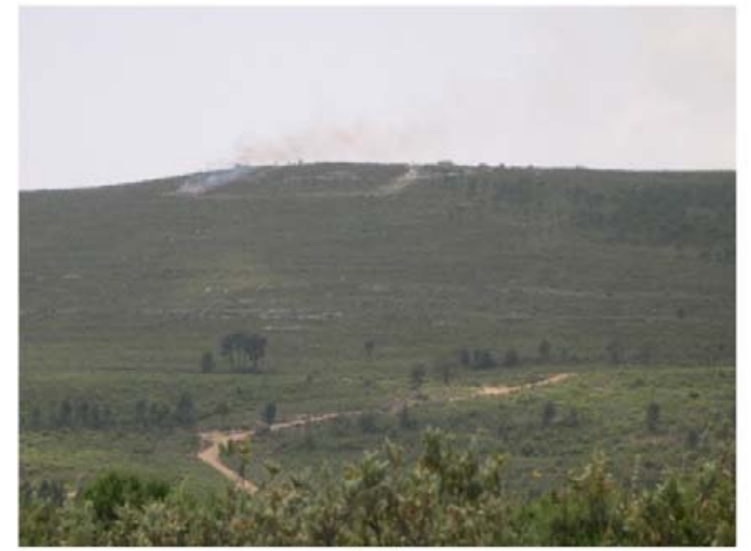

(a)

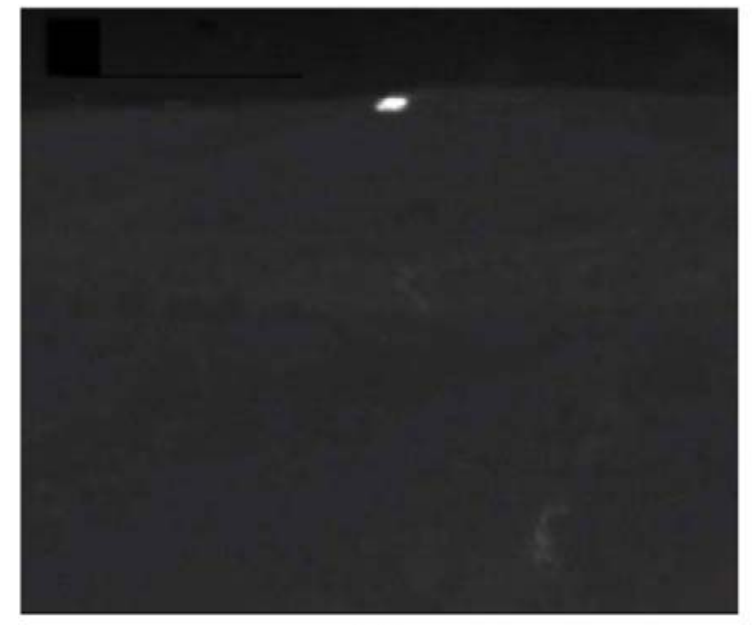

(c)

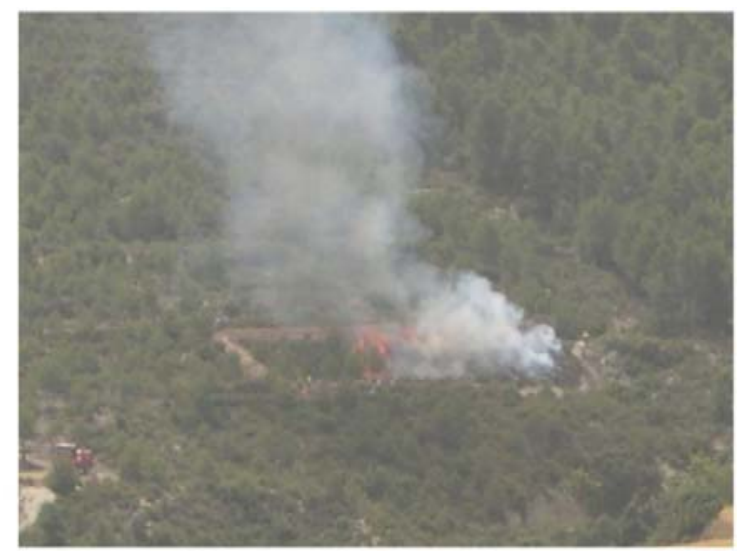

(b)

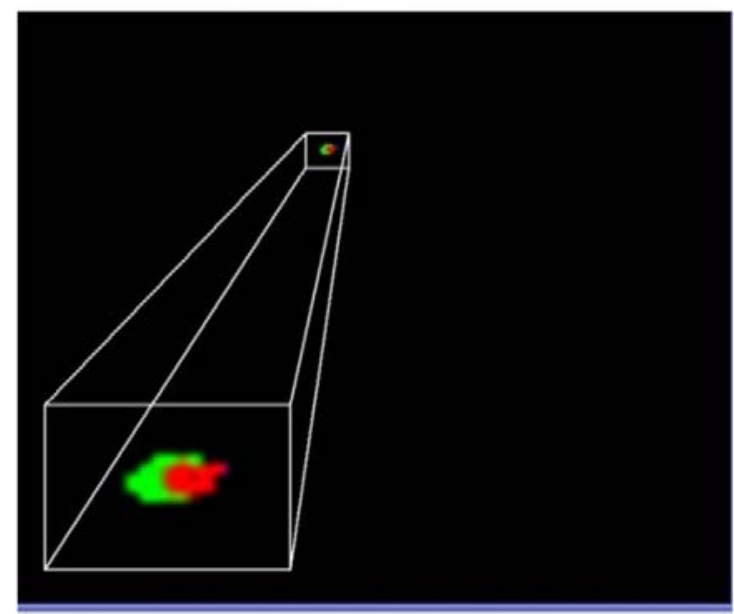

Persisting fire alarm Increasing fire alarm (d)

Figure 6. Visible images ( $a$ and $b$ ); infrared image (c); alarm representation captured from a controlled forest area $(d)$

Before putting the system into operation, we tested the system under real scenarios, by performing the capture of infrared images from real area-controlled forest fires under the supervision of several firemen (see the images in Figure 6).

Figure 6.c shows the corresponding alarms generated (a zoom has been done to improve visualization). We have represented the persistence alarm in green and the increase alarm in red. To generate a final alarm the two colours would overlap each other. The signal processing parameters were: $L=10, v=2, P_{\mathrm{f} 0}=P_{\mathrm{fi}}=10^{-3}$. In this example, the fire was detected at $1500 \mathrm{~m}$ of direct vision. It is important to note that the system has a distance range of $8 \mathrm{~km}$. Moreover, the operator can access this graphic alarm information remotely. Besides the control of $P_{\mathrm{ft}}$ (section 2.2), another important issue to take into account is the capability of the system to make an early detection of fire. 

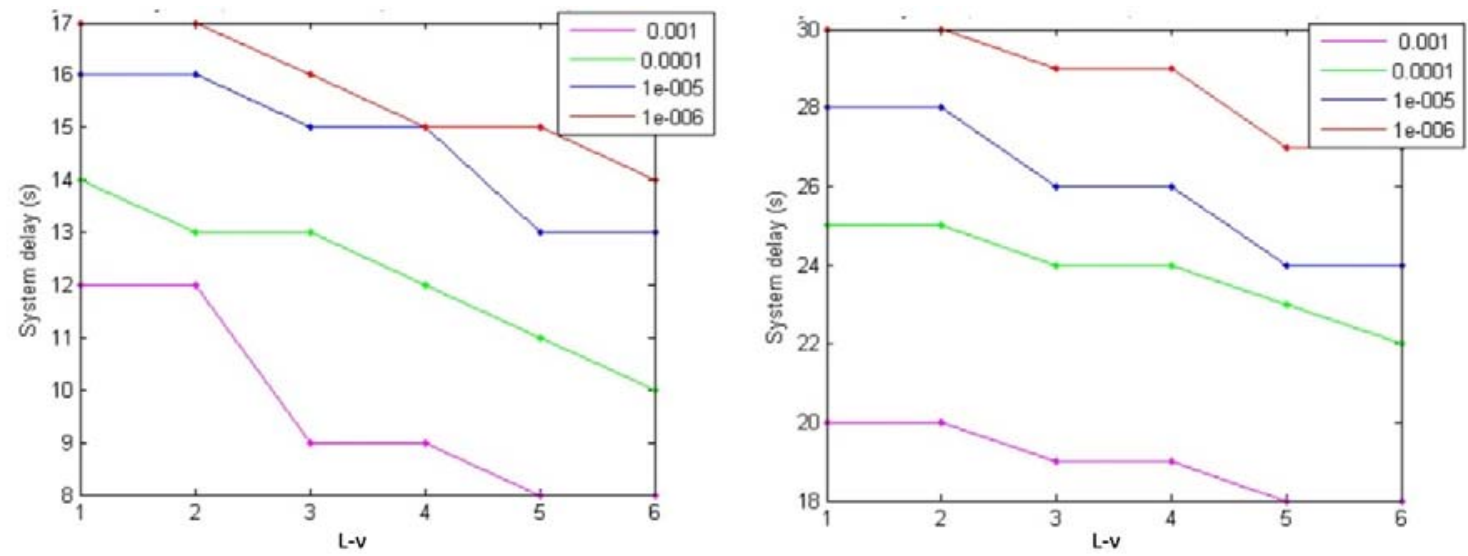

Figure 7. Delay of the system for early fire detection with different $P_{\mathrm{fo}} .(a) d=0,5 \mathrm{~km},(b) d=1,5 \mathrm{~km}$

The curves depicted in Figure 7 show the delay that the system requires to generate the first fire alarms from the beginning of the fire. These curves have been computed for a fixed $L=10$, and $P_{\mathrm{f} 0}, P_{\mathrm{fi}}$ varying from $10^{-3}$ to $10^{-6}$.

A comparison of the two graphics shown in Figure 7, indicates the following:

For a fixed value of $L$, if $v$ is decreased the persistence decision fusion is less restrictive and the fire can be detected earlier.

For a fixed value of $L-v$, if the $P_{\mathrm{f} 0}$ is increased, the system response speed increases.

If the distance between the camera and the fire, $d$, increases, the system delay increases.

For these two examples, (independently of the value of $d$ ), we can conclude that the system performs an earlier detection if we compare the system response with that of the average watchman.

The system has been in operation since July 2007. The system scans a total azimuth angle of $100^{\circ}$. Figure 8 shows a part of the system interface, which corresponds to 7 positions of the camera and their corresponding visual and infrared images. 

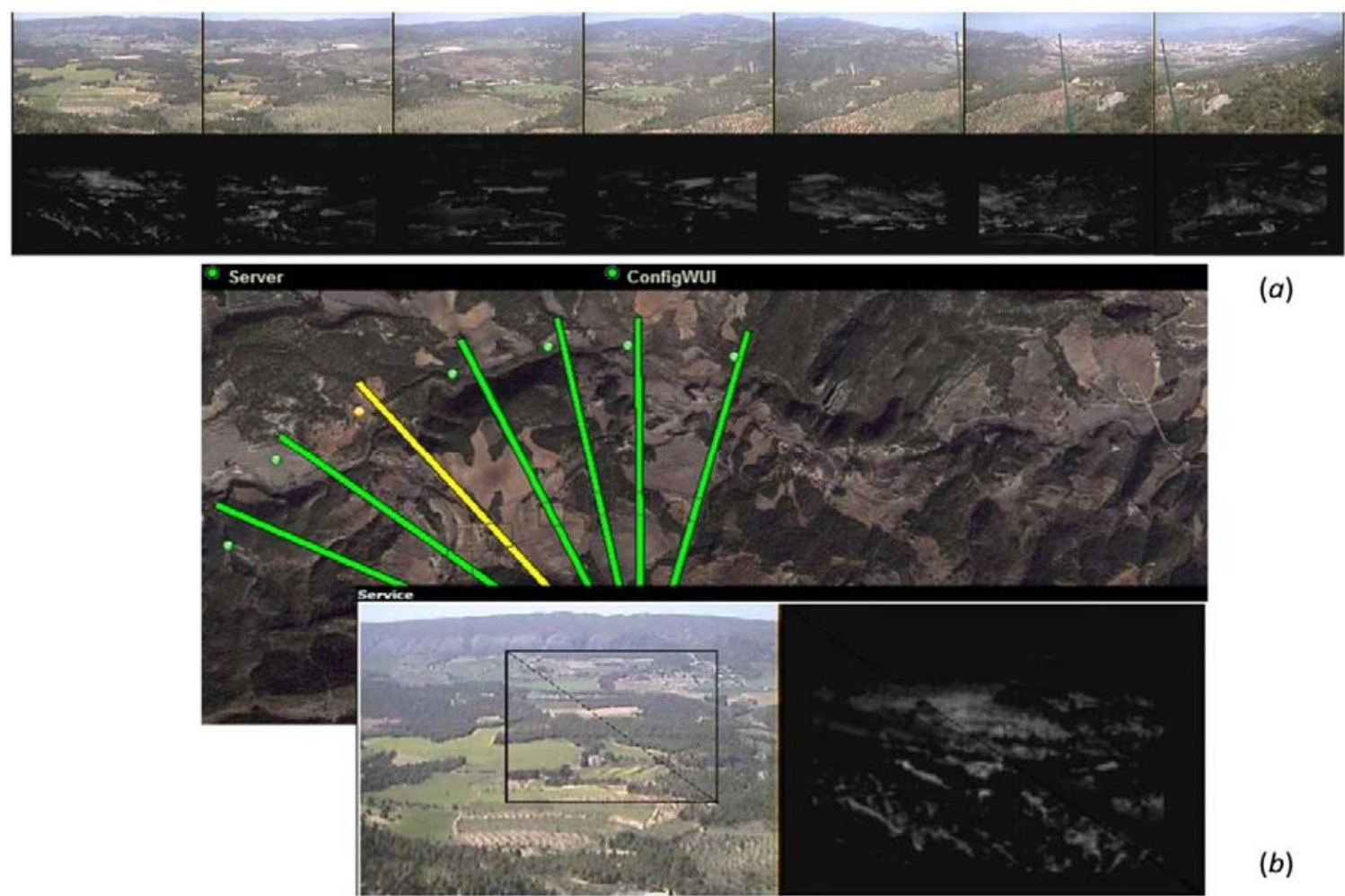

(a)

Figure 8. (a) System area of cover with 7 presets $(b)$ central station web application with access to one preset thermal and visible image

During its normal operation, the system has generated true detections and false alarms, as Figure 9 shows. A true detection is defined as an alarm that can be associated a posteriori to a true fire (whether or not the areas is under human control). On the other hand, a false alarm is one that cannot be associated to a real fire. 


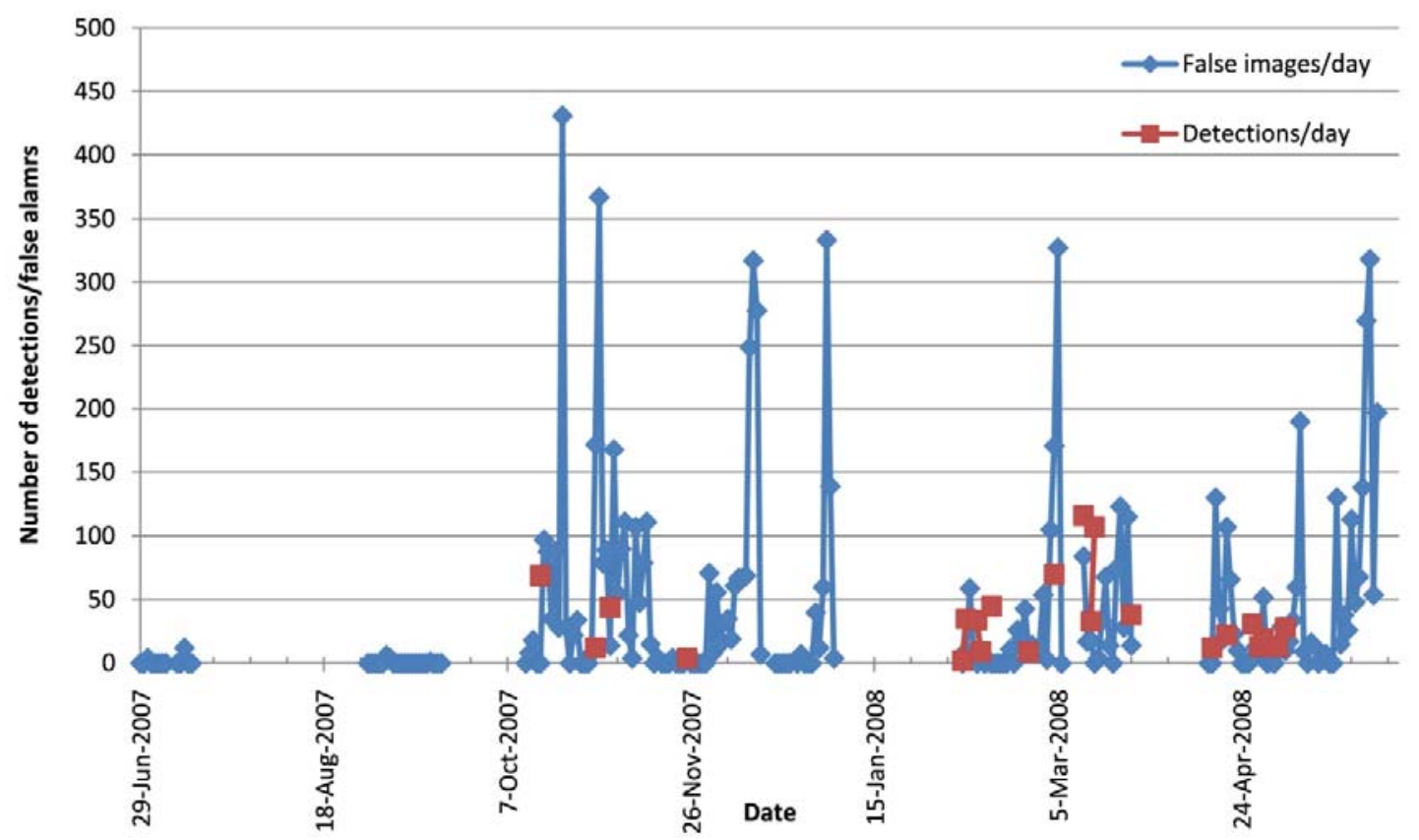

Figure 9. Temporary evolution of false alarms and detections per day

An average number of 40 false alarms per day were produced, which is in concordance with the configured value of $P_{\mathrm{ft}}$ of approximately $8 \times 10^{-9}$.

This number of alarms is very small especially taking into account that, throughout the day, one image is captured every 5.4 seconds on average (including the delays between presets). In a day, we have an average of 76,800 pixels per image ( 24 hours $\times 60$ minutes $\times 60$ seconds / 5.4 seconds $=16,000$ images/day with a resolution of $160 \times 120$ pixels $\times 4$ redundancy). Therefore, finally 16,000 images $\times 76,800$ pixels/image $=1,228,800,000$ pixels are being processed per day, 40 of which give false alarms on average.

Moreover, after a detailed analysis, some possible explanations can be found for the false alarms. Many of them were produced during the night or in especially cool weather conditions (for example, in the presence of fog). These results are due to the automatic calibration of the infrared camera. When the average infrared energy level is very low, the camera increases the sensitivity, so more false alarms are to be expected. Appropriate control of the automatic calibration of the camera by means of a temperature sensor could avoid most of these false alarms. Other false alarms were produced during the night by the lights of the city of Alcoy (part of the scanning angle included this city). Finally, some false alarms were observed on sunny days that had alternate periods of clouds.

Therefore, this number of false alarms can be drastically reduced when these problems are resolved, obtaining average values of 14 false alarms per day or a $P_{\mathrm{ft}}$ of $3 \mathrm{E}-9$. 
During this same period of time, the system has generated many true fire alarms (see example in Figure 10). Most of the true fires were detected from a significant distance approximately 1 to $10 \mathrm{~km}$. To our knowledge, no fires went undetected during this period.

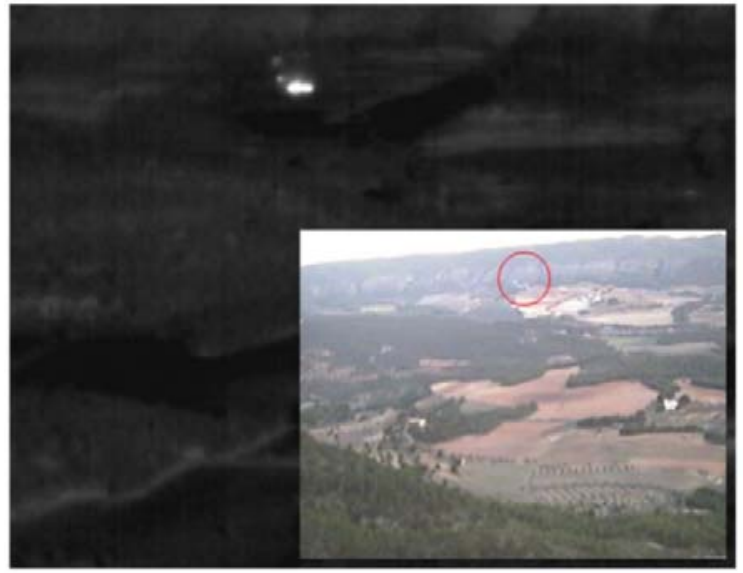

(a)

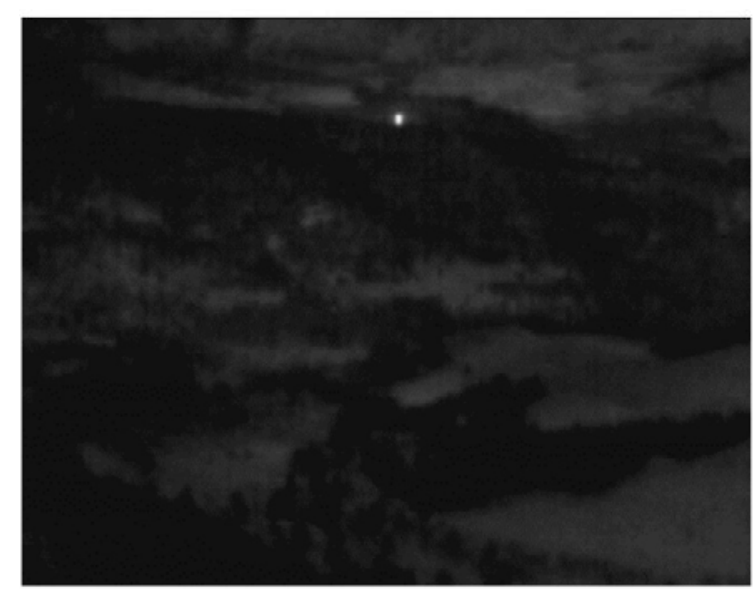

(c)

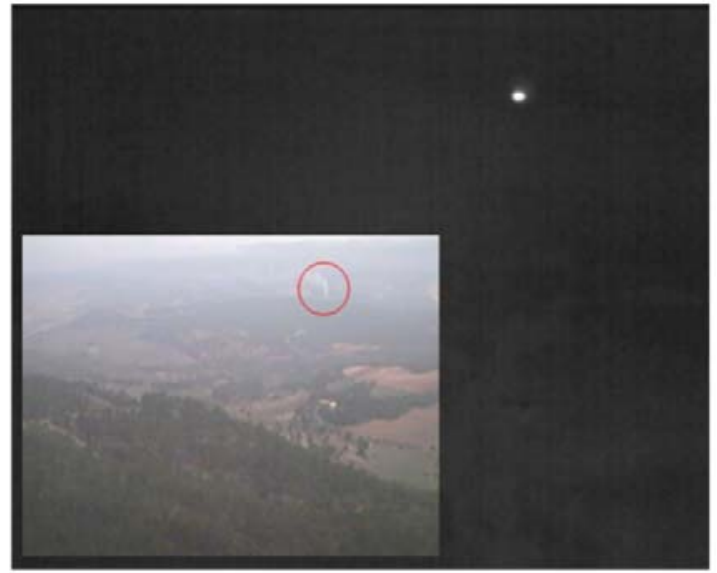

(b)

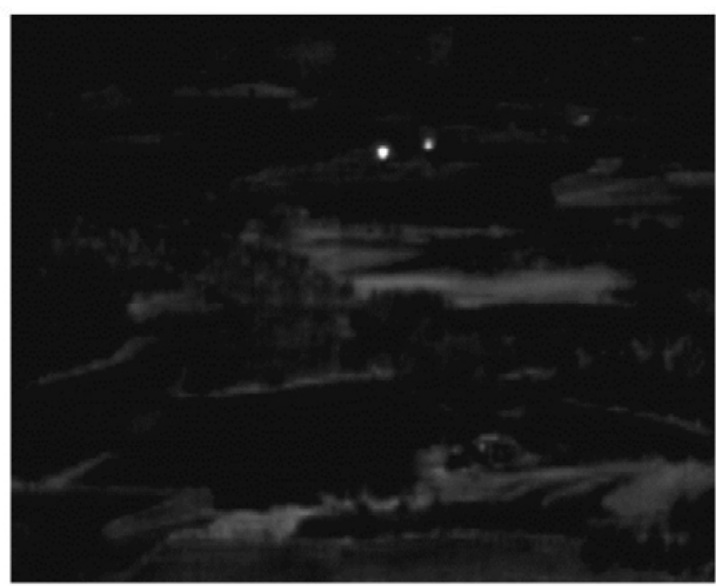

(d)

Figure 10. Four infrared images of detection examples in the operation period 2007-2008. (a), (b) infrared and visible images with a red mark of the detection example, $(c)$ and $(d)$ only infrared images of various detection examples

\section{Conclusions}

We have presented a ground remote automatic detection system for forest fire surveillance, which is applied to early fire detection based on thermal images obtained from infrared cameras. Advanced techniques, which are based on infrared signal processing, have been 
considered to determine the presence or absence of fire. We have used two different kinds of detectors that exploit different expected characteristics of a real fire, such as persistence at short-term and increase at long-term, in order to obtain a fusion of the decisions of each detector to be able to determine the presence or absence of fire.

The functionality of the system has been verified in diverse controlled real tests and in normal operation in order to authenticate the accuracy of the proposed system. We have shown the temporary evolution of false alarms and true detections to evaluate the performance of the system in the real environment.

The final PFA can be controlled by the appropriate selection of the detector parameters. We have also evaluated the delay of the system in order to generate an alarm corresponding to a real fire. The obtained results show great potential interest for signal processing-based systems of this kind to replace human surveillance.

\section{Acknowledgements}

This work has been supported by Generalitat Valenciana, under grant GVEMP06/001, and by MEC under FPU program.

\section{References}

ARRUE, B. C., OLLERO, A. and MARTINEZ DE DIOS, J.R., 2000, An intelligent System for False Alarm, Reduction in Infrared Forest Fire Detection. IEEE Intelligent Systems, 15, $64-73$.

BERNABEU, P, VERGARA, L., BOSCH, I., and IGUAL, J., 2004, A prediction/detection scheme for automatic forest fire surveillance. Digital Signal Processing, 14, $481-507$.

BOSCH, I., VERGARA, L., 2006, Forest Fire Detection by Infrared Data Processing. In Data Fusion for Situation Monitoring, Incident Detection and Response Management, 198 NATO Science Series, E. Shahbazian, G. Rovina, P. Valin (eds), , 931 - 944. (Computer \& Systems Sciences, IOS Press, 6)

BOSCH, I. GOMEZ, S. VERGARA, L. and MORAGUES, J. 2007, Infrared image processing and its application to forest fire surveillance. Proceedings of the AVSS 2007, IEEE Conference on Advanced Video and Signal Based Surveillance. 5-7 September 2007 (London, Great Britain), pp. 283 - 288.

BOSCH, I. GOMEZ, S. and VERGARA, L., 2007, Automatic Forest Surveillance Based on Infrared Sensors; Proceedings of the SensorComm 2007. International Conference on Sensor Technologies and Applications, 14-20 October 2007 (Valencia, Spain), pp.572 577.

BRIZ, S., DE CASTRO, J. A., ARANDA, J.M., MELÉNDEZ, J. and LÓPEZ, F., 2003, Reduction of false alarm rate in automatic forest fire infrared surveillance systems. Remote Sensing of Environment, 86, 19 - 29. 
DEDEOGLU, Y., TOREYIN, B., Güdkübay, U., ENIS, A. and ETIN, C., 2005, RealTime fire and flame detection in video. IEEE 30th International Conference on Acoustics, Speech and Signal Processing (ICASSP), $669-672$.

LI, J., QI, Q., ZOU, X., PENG, H., JIANG, L. and LIANG, Y., 2005, Technique for automatic forest fire surveillance using visible light image. Proc. on the IGARSS, 5, 3135 3138.

PASTOR, E., ZÁRATE, L., PLANAS, E. and ARNALDOS, J., 2003, Mathematical models and calculation systems for the study of wildland fire behaviour. Prog Energy Combust Sci, 29, 139 - 152.

VERGARA, L. and BERNABEU, P., 2000, Automatic Signal Detection applied to fire control by infrared digital signal processing. Signal Processing, 80, 659 - 669.

VERGARA, L. and BERNABEU, P., 2001, Simple approach to nonlinear prediction. Electronic Letters, 37, 926 - 928.

VICENTE, J. and GUILLEMANT, P., 2002, An image processing technique for automatically detecting forest fire. Int. J. Therm. Sci.41. $113-1120$. 